\title{
Treatment of oral mucositis pain following radiation therapy for head-and-neck cancer using a bioadhesive barrier-forming lipid solution
}

\author{
Tatiana Hadjieva • Eva Cavallin-Ståhl • \\ Margareta Linden $\cdot$ Fredrik Tiberg
}

Received: 5 September 2013 / Accepted: 1 January 2014 / Published online: 18 January 2014

(C) The Author(s) 2014. This article is published with open access at Springerlink.com

\begin{abstract}
Purpose CAM2028, a vehicle that forms a bioadhesive lipid barrier when applied to the oral mucosa, was developed as a carrier system for local delivery of benzydamine, an NSAID used for pain relief in oral mucositis. This trial compared the analgesic effect of CAM2028 plus benzydamine (CAM2028benzydamine) with unmedicated CAM2028 (CAM2028control) for the treatment of oral mucositis in patients with head-and-neck cancer.

Methods Thirty-eight study participants were enrolled during their 3rd to 4th week of radiation therapy. Participants were required to have symptomatic oral mucositis (WHO Grade 2 or above) at screening and pain scores of at least 6 on an 11point Likert scale at screening and on each day before treatment with study medication. After undergoing radiation, patients were administered a single dose of CAM2028-control or CAM2028-benzydamine 2 days apart, in a randomized crossover fashion. Pain was assessed over the following $8 \mathrm{~h}$.

Results With both treatments, patients experienced a mean $40 \%$ decrease in pain intensity at $6 \mathrm{~h}$ (the primary study endpoint). Both treatments resulted in significant pain relief within $5 \mathrm{~min}$ of application that was evident during the entire 8-h assessment period. There was no difference in pain relief between the two interventions at any time point. Both treatments were safe and well tolerated.

Conclusions CAM2028-benzydamine and CAM2028control were both efficacious in reducing pain in patients with
\end{abstract}

\section{T. Hadjieva}

Department of Radiotherapy, Medical University, Sofia, Bulgaria

E. Cavallin-Ståhl

Division of Oncology, Lund University Hospital, Lund, Sweden

M. Linden $\cdot$ F. Tiberg $(\bowtie)$

Camurus AB, Ideon Science Park, Sölvegatan 41, 22370 Lund,

Sweden

e-mail: fredrik.tiberg@camurus.com oral mucositis related to radiation therapy for head-and-neck cancer. Analgesic effects of both medications were immediate, clinically significant, and persistent for up to $8 \mathrm{~h}$.

Keywords Oral mucositis · Head-and-neck neoplasms · Analgesia $\cdot$ Benzydamine $\cdot$ Crossover studies $\cdot$ Treatment . Rinses $\cdot$ Coating agents $\cdot$ Radiation

\section{Introduction}

Oral mucositis has emerged as one of the most troublesome consequences of current high-intensity cancer treatment regimens. High-grade (grade 3 or 4 ) oral mucositis affects virtually $100 \%$ of patients who undergo high-dose radiation therapy for head-and-neck cancer and is a major limiting factor for advanced chemoradiation regimens $[1,2]$. Oral mucositis has a significant negative effect on the patient experience of cancer therapy, contributing to difficulty eating and drinking, weight loss, fatigue, pain, sleep deprivation, and functional impairment [3]. Intolerable oral mucositis is the primary cause of unplanned treatment breaks in head-andneck cancer, limiting the efficacy of aggressive treatment regimens and negatively affecting treatment outcomes $[4,5]$.

Oral mucositis is associated with greater health care resource use and higher economic costs due to patients' need for supportive care, parenteral or enteral nutrition, opioid analgesics, and hospitalization [6, 7]. According to one analysis in patients with head-and-neck cancer, oral mucositis was associated with an additional treatment cost of $\$ 1,700$ to $\$ 6,000$, depending on the grade [2]. In another study, high-grade oral mucositis in patients with head-and-neck cancer was associated with an incremental treatment cost of $\$ 17,000$ (in 20032005), largely driven by inpatient hospitalization to support alimentation [8]. 
Recommended evidence-based management of oral mucositis in patients with head-and-neck cancer consists of implementing an oral care protocol, regularly assessing patients for oral symptoms, and empiric short-term analgesics [1, $9,10]$. Gentle brushing and flossing and the use of bland oral rinses are common features of oral care protocols. Although a number of interventions are available to relieve pain associated with oral mucositis, there is currently a lack of wellsupported therapies. According to a recent Cochrane review, [11] randomized clinical trials of oral mucositis treatments are few and offer little clinical guidance. The existing evidence has not shown mouthwashes containing analgesics or antiseptics to be superior to placebo or to unmedicated preparations. Behavioral approaches - relaxation and imagery or hypnosis-were also not superior to control treatments. There is a need for effective, rapid-acting treatment that can be easily deployed in the clinic as needed.

Benzydamine is among the agents frequently recommended for pain relief in oral mucositis in patients undergoing moderate-dose radiation therapy for head-and-neck cancer $[1,9,10]$. Benzydamine is a nonsteroidal antiinflammatory drug with a long history of safe use in patients. Results of clinical trials of benzydamine in oral mucositis have been mixed: two studies showed no greater effects on pain than placebo [11] but one trial suggests it is effective in preventing mouth ulcers and delaying the use of systemic analgesics following radiation for head-and-neck cancer [12]. The currently available oromucosal benzydamine products have a short duration of effectiveness and need to be administered every 1.5 to $3 \mathrm{~h}$.

The present report describes the development and pilottesting of a new formulation of benzydamine designed to extend its analgesic effects for a sustained period. The pilot study described herein, a randomized crossover trial in patients with head-and-neck cancer, had the unexpected result that the formulation itself had a prolonged analgesic effect, regardless of whether it contained benzydamine or was unmedicated. Based on these and other data, and by virtue of its mechanical mode of action, the formulation, CAM2028-control (episil ${ }^{\circledR}$, Camurus) has recently received FDA and EU approvals as a medical device for the management of pain in oral mucositis.

\section{Development of investigational formulation}

CAM2028-benzydamine was developed as a lipid-based drug carrier system for local and extended delivery of benzydamine in the oral cavity. After application to the oral mucosa, phospholipid and triglyceride lipid components of the formulation spread in the oral cavity and self-assemble with a trace volume of aqueous fluid at the mucosal surface to form a bioadhesive liquid crystalline lining protecting the sore and inflamed mucosa, including exposed nerve endings (Fig. 1). The liquid crystalline film formation occurs by molecular self-assembly of the lipid components and ambient water molecules present in saliva, and does not involve any chemical reaction. The film adheres to the palate, the inside of the cheeks, gums, and the rim of the tongue. In the formulation containing benzydamine, the drug was slowly released into the oral mucosa, with the aim of conferring rapid-onset and prolonged analgesia. Additional effects of the bioadhesive lipid formulation may include lubrication and mechanical protection of the sore mucosa and possibly a moistening effect.

The present study was conducted with the primary objective of testing the analgesic effect of CAM2028 with benzydamine compared with CAM2028 without benzydamine (the FDA approved prescription formulation of episil $^{\circledR}$ ) over an 8-h period. An additional objective was to assess the safety and tolerability of a single dose of the new formulation.

\section{Patients and methods}

CAM2028, with and without benzydamine, was evaluated in a crossover, double-blind, placebo-controlled, single-dose, randomized, proof-of-concept trial in head-and-neck cancer patients receiving radiotherapy. The study was conducted at five oncology centers in Bulgaria. The protocol and the statement of informed consent were approved in Bulgaria by an Independent Ethics Committee. The trial was conducted in accordance with the Declaration of Helsinki and its revisions as well as with the valid local and national laws of Bulgaria; with the International Conference on Harmonisation (ICH) Harmonised Tripartite Guideline for Good Clinical Practice (E6) issued in July 1996; and with the relevant European Commission Directives. All patients gave written informed consent prior to enrollment.

Study participants were male or female patients, at least 18 years of age, undergoing outpatient radiation therapy for newly diagnosed head-and-neck cancer. At screening, all patients had undergone radical or postoperative radiotherapy to a significant part of clinically visible oral and/or pharyngeal mucosa at two or more anatomic sites. The trial began during weeks 3 to 4 of radiotherapy and took place over a maximum duration of 12 days. After a screening period of up to 7 days, treatment was randomly assigned; at this point, patients must have received at least one third of the planned total dosage of radiation. At screening, participants were required to exhibit symptomatic oral mucositis (WHO grade 2 or above). Pain scores of at least 6 on a Likert scale of 0 to 10 were required at screening and before each treatment. Clinically relevant exclusion criteria were pregnancy or breastfeeding, known contraindications or hypersensitivity to the trial drug or other NSAIDs, immunocompromised, hepatitis A or B infection, 


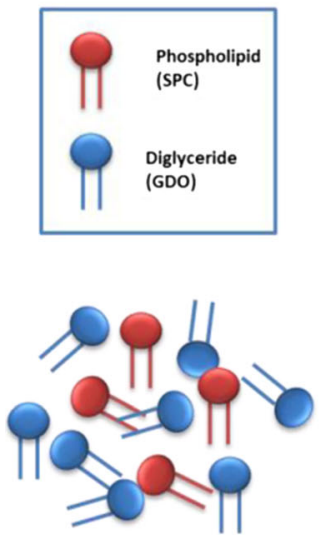

Lipid solution
CAM2028

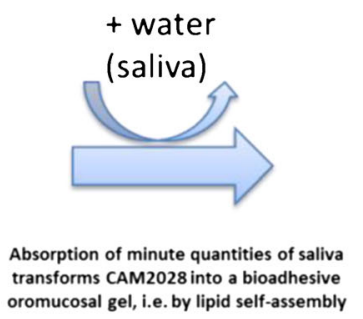
transforms CAM2028 into a bioadhesive
oromucosal gel, i.e. by lipid self-assembly
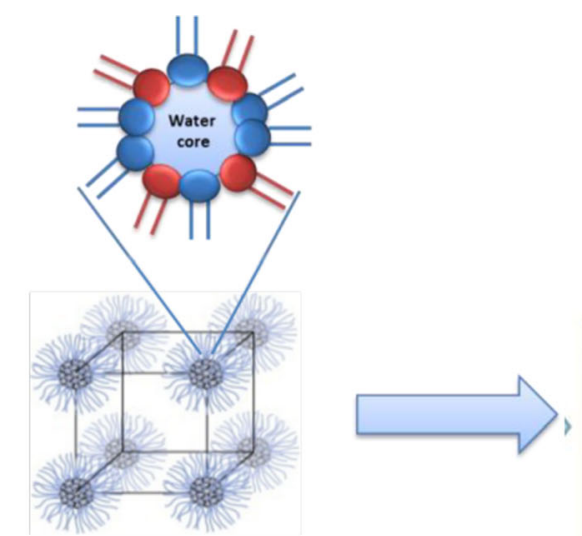

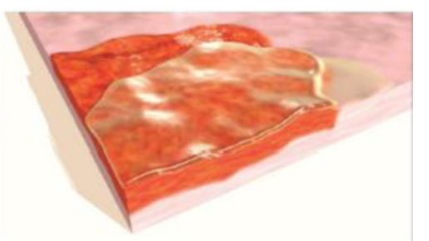

Reversed micellar cubic phase $\left(I_{11}\right)$

Fig. 1 Mechanism of action of CAM2028. After application to the oral mucosa, phospholipid and triglyceride lipid components self-assemble with a trace volume of water (saliva) to form a bioadhesive and protective liquid crystalline lining of the oral mucosa

and prior chemotherapy, immunotherapy, or radiation to the upper airways.

\section{Study design and procedures}

Eligibility was confirmed at the screening visit, a maximum of 7 days before the first treatment visit. Patients were treated with randomized study medication on Treatment Days 1 and 3 , and returned for a final follow-up evaluation on Day 5. At the first treatment visit, each patient was randomly allocated to one of two sequences: CAM2028-benzydamine (Day 1) followed by CAM20208-control (Day 3), or CAM2028control followed by CAM2028-benzydamine. Patients were assigned a random number and received trial medication sent from the study coordinating center with the corresponding number. The list of random numbers was generated at the coordinating center using the permuted bloc method based on SAS ${ }^{\circledR}$ for Windows (SAS Institute Inc., Cary, NC, USA). Treatment allocation was concealed from the investigators, staff at the trial sites, trial monitors, data analysts and managers, and patients.

Patients were administered the trial medication after undergoing radiotherapy. The investigational medication consisted of the oromucosal solution CAM2028 containing benzydamine $28.2 \mathrm{mg} / \mathrm{mL}$. The comparison treatment was CAM2028-control. One milliliter of either the study medications was applied to the oral mucosa using a syringe, and patients were instructed to swirl the medication around in the mouth for approximately $15 \mathrm{~s}$ and then spit out any residual medication. The procedure was repeated after $5 \mathrm{~min}$.

Concomitant use of any oromucosal medication was not permitted from 3 days before dosing on Day 1 until the final evaluation on Day 5. This prohibition included oromucosal analgesics, anti-inflammatories, antimicrobials, and mucoprotective agents. Also prohibited were systemic antiinflammatories, analgesics, and drugs that may affect the process of oral mucositis, including growth factors, vitamins, and newly introduced steroids. Immediate-release paracetamol, opioids, and opiates were permitted as rescue medication. Eating and drinking hot beverages was not allowed until $3 \mathrm{~h}$ after drug administration.

\section{Evaluations}

On each treatment day, oromucosal pain was assessed by the patient using an 11-point Likert scale $(0=$ no pain, $10=$ worst possible pain). Patients recorded their pain scores before dosing and at 5 and $30 \mathrm{~min}$ and 1,2,3,6, and $8 \mathrm{~h}$ post-dose. After the initial 3-h assessment, patients were allowed to leave the hospital with a diary for recording pain scores at 6 and $8 \mathrm{~h}$.

Adverse events were to be recorded throughout the study period, not just on treatment days. Symptomatic changes were elicited from the patient in a non-leading manner at each visit. Clinically relevant laboratory values or pathological changes, as determined by the investigator, were also to be recorded as adverse events. Investigators were to record information on an Adverse Event Report Form, including severity grading, resolution, and relationship to study medication as determined by the investigator. An adverse event was defined as treatmentemergent if it had onset or worsened after the first use of study medication and no more than 1 day after the last use.

\section{Statistical methods}

A total of 38 patients were enrolled with the aim of a final sample of 32 evaluable patients. As this was a pilot study, sample size was determined empirically. The primary efficacy 
endpoint was the pain intensity difference (PID), calculated as the change in the Likert pain scale from baseline to $6 \mathrm{~h}$ after administration of study medication. Secondary outcome measures were the PID at other time intervals, peak pain (maximum Likert score on each treatment day), and the area under the curve (AUC) of the PID. The last observation carried forth (LOCF) method was used to replace missing pain score data from the same day. The primary efficacy endpoint was evaluated using the mixed effects analysis of covariance (ANCOVA) model on ranks. The model included effects for treatment, center, sequence, period, patient, and baseline Likert pain score. Secondary endpoints were analyzed in the same manner. Analyses were planned on the safety data set (patients who received at least one dose of study medication), the full data set (patients who provided data on the primary efficacy variable for both treatments) and the perprotocol data set (patients who completed the trial with no protocol deviations). Analyses were conducted using SAS ${ }^{\circledR}$ release 8.2.

\section{Results}

Patient characteristics and disposition

Thirty-eight patients were randomly assigned to treatment, and all completed the trial. Twenty patients were assigned to receive CAM2028-benzydamine on Day 1 and CAM2028control on Day 3, and 18 patients received the treatments in the opposite order. All were included in the safety population and the full data set. Three patients were not included in the per-protocol set because they had a baseline pain score lower than 6 on the second treatment visit (when all three were scheduled to receive CAM2028-benzydamine) and three others had minor protocol violations in recording their pain scores. The per-protocol set consisted of 19 patients who received the CAM2028-benzydamine first and 13 who received CAM2028-control first. All patients took all doses of study medication.

Demographic characteristics did not differ between the two treatment groups, with the exception that women were overrepresented in the group receiving placebo first (5 versus 1). Patients were a median age of 52 years (range 32 to 73), 32 patients $(84.2 \%)$ were male, and all were of Caucasian ethnicity. Mean body mass index was $21.5 \mathrm{~kg} / \mathrm{m}^{2}$. Nearly half of the participants were sporadic consumers of alcohol (47.4\%), rather than non-drinkers $(26.3 \%)$ or regular drinkers (26.3\%). Most were current smokers (42.1\%) or ex-smokers $(42.1 \%)$. Patients were screened for study participation at a median of about 6 weeks following the diagnosis of head-andneck cancer (range 9 days to $>17$ weeks).
Treatment efficacy

We present results of the analysis of treatment efficacy for the per-protocol data set; results in the full patient population did not differ. The mean pain intensity difference at $6 \mathrm{~h}$ was 2.5 for CAM2028-benzydamine and 2.2 for CAM2028-control (i.e., patients experienced an average 2-plus-point decline in pain intensity from baseline to $6 \mathrm{~h}$ after treatment). Mean pain intensity ratings decreased by about $40 \%$, from a baseline of 6.5 (CAM2028-benzydamine) or 6.4 (CAM2028-control) to 4.6 (both treatments) already within 5 min of study medication application and remained at this level or lower throughout the $8 \mathrm{~h}$ of observation (Table 1, Fig. 2). At no time did mean pain ratings or PID differ statistically between the two treatments.

The mean AUC of pain intensity over time did not differ between the two treatments. The mean peak PID was 2.8 for CAM2028-benzydamine and 2.7 for CAM2028-control. All of the analyses of pain intensity outcomes showed a statistically significant clinical center effect, with one center reporting larger PID values than others. The data do not suggest any explanation for this difference.

\section{Safety and tolerability}

Four patients experienced seven treatment-emergent adverse events. None were considered severe, and none were judged to be related to study medication. Two patients experienced nausea or vomiting on CAM2028-benzydamine, one on CAM2028-control, and one on both treatments. Upper respiratory tract infection and hemoptysis each occurred in 1 patient who received the CAM2028-benzydamine.

Regarding rescue medication, only paracetamol was used in a total of 8 patients $(21 \%)$ during the course of the treatment period. None of the patients used opioids as rescue medication.

Table 1 Patient evaluations of pain intensity on an 11-point Likert scale, before and at selected time points after administration of CAM2028benzydamine or CAM2028-control (per-protocol population, $n=32$ )

\begin{tabular}{|c|c|c|c|c|c|}
\hline & $\begin{array}{l}\text { Before } \\
\text { dosing }\end{array}$ & $5 \min$ & $1 \mathrm{~h}$ & $6 \mathrm{~h}$ & $8 \mathrm{~h}$ \\
\hline \multicolumn{6}{|c|}{ CAM2028-benzydamine } \\
\hline Mean \pm SD & $6.5 \pm 0.80$ & $4.6 \pm 1.60$ & $4.1 \pm 1.98$ & $4.0 \pm 2.06$ & $4.2 \pm 2.03$ \\
\hline Median & 6.0 & 4.5 & 4.0 & 4.0 & 4.0 \\
\hline Range & $6-8$ & $2-8$ & $1-8$ & $1-8$ & $1-8$ \\
\hline \multicolumn{6}{|c|}{ CAM2028-control } \\
\hline Mean \pm SD & $6.4 \pm 0.72$ & $4.6 \pm 1.54$ & $3.9 \pm 2.0$ & $4.3 \pm 2.23$ & $4.4 \pm 2.09$ \\
\hline Median & 6 & 4 & 4 & 4 & 4 \\
\hline Range & $6-8$ & $1-8$ & $0-8$ & $0-9$ & $0-9$ \\
\hline
\end{tabular}




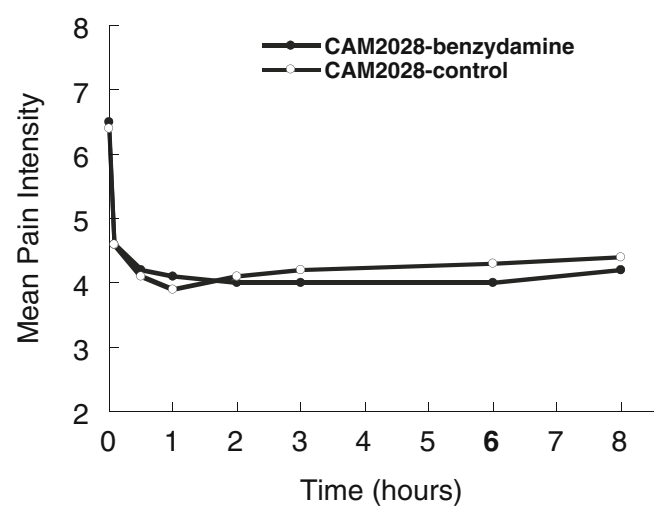

Fig. 2 Patient evaluations - pain intensity (11 point Likert scale) versus time after application of study medication at time 0 (per-protocol set, $N=32$ )

\section{Discussion}

This randomized trial set out to compare the effects CAM2028-benzydamine and CAM2028-control in patients who experienced oral pain while undergoing radiation therapy for head-and-neck cancers. Both study medications had an immediate and clinically significant pain-relieving effect within $5 \mathrm{~min}$ of application and that analgesic effect was maintained for up to $8 \mathrm{~h}$. We found that the two treatments had similar effects in reducing pain, due to the efficacy of the bioadhesive and physically protective mechanical barrier, which we had not predicted. The two treatments did not differ on the main outcome measure, PID from baseline, or on any other measure of pain.

Based on subsequent experience, administration of the currently approved CAM2028 product (episil@, Camurus) differs from that described in this study. A phase 2 clinical trial showed that upon application, the formulation takes less than $1 \mathrm{~min}$ to form a bioadhesive and protective lining and that a smaller quantity of CAM2028 is sufficient to provide effective coverage of oromucosal surfaces, rather than the $1 \mathrm{~mL}$ used in the present study, where patients were informed to spit out excess solution [13]. The current product (episil@) labeling instructs patients to spray a $0.15-\mathrm{mL}$ metered dose of the liquid into the oral cavity 1 to 3 times (delivering a quantity ranging from 0.15 to $0.45 \mathrm{~mL}$ ) and to allow $5 \mathrm{~min}$ for the bioadhesive barrier to form. They are then able to eat or drink immediately, rather than waiting several hours.

The similar treatment effects of CAM2028 with or without benzydamine suggest that benzydamine did not contribute additionally to the reduction of oral mucositis pain compared with the unmedicated CAM2028-control. Although no further effects were seen with benzydamine, it cannot be excluded that benzydamine has a role in the management of oral mucositis, but this would require further investigation in repeat dose studies. However, the present results obtained with single doses of CAM2028, with and without benzydamine, suggest that the observed analgesic effect is mainly due to the effective mechanical protection of the oral mucosal surfaces provided by the in situ barrier-forming lipid solution. This lipid barrier covers and isolates the sore mucosa and exposed nerve endings from external irritation. Thus, CAM2028 offers a unique approach for treating local pain associated with oral mucositis that does not interfere with the use of other therapies and that, additional experience suggests, may be used with additive effects. In a preliminary study in patients undergoing highdose chemotherapy for autologous stem-cell transplantation, CAM2028 combined with cryotherapy resulted in fewer and less severe occurrences of oral mucositis compared with cryotherapy alone [14]. Unlike cryotherapy alone, CAM2028 also spared patients the need for total parenteral nutrition and for opioids for pain relief.

At present, there are few other effective treatments for pain associated with oral mucositis. The most widely prescribed topical therapy is a pharmacist-compounded mouthwash generally called "magic" or "miracle mouthwash" $[15,16]$. There is no standard formulation for this mouthwash, but the most popular formulation consists of viscous lidocaine, diphenhydramine, and an antacid. Although its widespread use suggests clinicians perceive it to be effective, there is no clinical trial evidence supporting the efficacy of compounded mouthwash in relieving pain associated with oral mucositis [11], and one trial found it no more effective than salt and soda [17]. Because lidocaine-containing products can cause numbness, patients are advised to avoid eating or performing oral hygiene measures after using these products to avoid accidental trauma to oral tissues [9]. Lidocaine can also dampen the gag reflex, placing the patient at risk for aspiration. The diphenhydramine component of the mouthwash formulation can exacerbate the xerostomia that patients with oral mucositis frequently experience.

Based on the results of this study in 38 cancer patients with oral mucositis while undergoing radiation treatment for headand-neck cancer, it is concluded that CAM2028 resulted in immediate and significant pain relief with a duration that was maintained for up to $8 \mathrm{~h}$. The reduction of pain was similar between both CAM2028 treatments, suggesting that benzydamine did not contribute additionally to the reduction of oral mucositis pain compared with the CAM2028-control. Both CAM2028 treatments were found to be safe and well tolerated in this group of patients.

Acknowledgments We thank the following study investigators: Vaselina Parvanova MD, $\mathrm{PhD}$, Marianna Yaneva, $\mathrm{MD}, \mathrm{PhD}$, Raumen Gabrovski, MD, $\mathrm{PhD}$, and Petio Chilingirov, $\mathrm{MD}, \mathrm{PhD}$ for their contributions to the study. We also thank Jackie Turner at Premier Research plc for performing biostatistical analysis.

Conflict of interest This study was funded by Camurus AB, Lund, Sweden. Two of the authors (ML and FT) are employees and principals of Camurus. The others have no financial relationships to disclose. 
The authors have full control of all primary data and have agreed to allow the journal to review the data if requested.

Open Access This article is distributed under the terms of the Creative Commons Attribution Noncommercial License which permits any noncommercial use, distribution, and reproduction in any medium, provided the original author(s) and the source are credited.

\section{References}

1. Peterson DE, Bensadoun RJ, Roila F, ESMO Guidelines Working Group (2008) Management of oral and gastrointestinal mucositis: ESMO clinical recommendations. Ann Oncol 19(Suppl 2):122-125. doi:10.1093/annonc/mdn109

2. Elting LS, Cooksley CD, Chambers MS, Garden AS (2007) Risk, outcomes, and costs of radiation-induced oral mucositis among patients with head-and-neck malignancies. Int J Radiat Oncol Biol Phys 68(4):1110-1120

3. Elting LS, Keefe DM, Sonis ST, Garden AS, Spijkervet FK, Barasch A, Tishler RB, Canty TP, Kudrimoti MK, Vera-Llonch M, Burden of Illness Head and Neck Writing Committee (2008) Patient-reported measurements of oral mucositis in head and neck cancer patients treated with radiotherapy with or without chemotherapy: demonstration of increased frequency, severity, resistance to palliation, and impact on quality of life. Cancer 113(10):2704-2713

4. Russo G, Haddad R, Posner M, Machtay M (2008) Radiation treatment breaks and ulcerative mucositis in head and neck cancer. Oncologist 13(8):886-898. doi:10.1634/theoncologist.2008-0024

5. Rosenthal DI (2007) Consequences of mucositis-induced treatment breaks and dose reductions on head and neck cancer treatment outcomes. J Support Oncol 5(9 Suppl 4):23-31

6. Murphy BA (2007) Clinical and economic consequences of mucositis induced by chemotherapy and/or radiation therapy. J Support Oncol 5(9 Suppl 4):13-21

7. Silverman S Jr (2007) Diagnosis and management of oral mucositis. J Support Oncol 5(2 Suppl 1):13-21

8. Nonzee NJ, Dandade NA, Patel U, Markossian T, Agulnik M, Argiris A, Patel JD, Kern RC, Munshi HG, Calhoun EA, Bennett CL (2008)
Evaluating the supportive care costs of severe radiochemotherapyinduced mucositis and pharyngitis : results from a Northwestern University Costs of Cancer Program pilot study with head and neck and nonsmall cell lung cancer patients who received care at a county hospital, a Veterans Administration hospital, or a comprehensive cancer care center. Cancer 113(6):1446-1452

9. Bensinger W, Schubert M, Ang KK et al (2008) NCCN Task Force Report. Prevention and management of mucositis in cancer care. J Natl Compr Cancer Netw 6(Suppl 1):S1-S21, quiz S22-24

10. Keefe DM, Schubert MM, Elting LS et al (2007) Mucositis study section of the multinational association of supportive care in cancer, international society for oral oncology. Updated clinical practice guidelines for the prevention and treatment of mucositis. Cancer 109(5):820-831. doi:10.1002/cncr.22484

11. Clarkson JE, Worthington HV, Furness S, McCabe M, Khalid T, Meyer S (2010) Interventions for treating oral mucositis for patients with cancer receiving treatment. Cochrane Database Syst Rev 8, CD001973. doi:10.1002/14651858.CD001973.pub4

12. Epstein JB, Silverman S Jr, Paggiarino DA, Crockett S, Schubert MM, Senzer NN, Lockhart PB, Gallagher MJ, Peterson DE, Leveque FG (2001) Benzydamine $\mathrm{HCl}$ for prophylaxis of radiation-induced oral mucositis: results from a multicenter, randomized, double-blind, placebo-controlled clinical trial. Cancer 92(4):875-885

13. Barauskas J, Christerson L, Wadsäter M, Lindström F, Lindqvist AK, Tiberg F (2014) Bioadhesive lipid compositions: self-assembly structures, functionality and medical applications. Mol Pharm. doi:10.1021/mp400552u

14. Svanberg A, Birgegård G, Öhrn K (2010) A new preventive strategy using a bioadhesive oral mucosal lipid solution and oral cryotherapy to protect the oral cavity and reduce the need for total parenteral nutrition (TPN) for patients undergoing autologous stem cell transplantation (Abstract no. 08-076). Support Care Cancer 18(Suppl 3):S114-S115

15. Tom WC (2007) Magic mouthwash: an update. Pharm Lett Prescr Lett 23:1-5

16. Sonis S (2011) The quest for effective treatments of mucositis. J Support Oncol 9(5):170-171. doi:10.1016/j.suponc.2011.07.001

17. Dodd MJ, Dibble SL, Miaskowski C, MacPhail L, Greenspan D, Paul SM et al (2000) Randomized clinical trial of the effectiveness of 3 commonly used mouthwashes to treat chemotherapy-induced mucositis. Oral Surg Oral Med Oral Pathol Oral Radiol Endod 90(1):39-47 